Journal of

\section{Applied Crystallography}

ISSN 0021-8898

\section{Philips Analytical X'Celerator}

Philips Analytical announces a new fast $\mathrm{X}$-ray detector, the $\mathbf{X}$ 'Celerator, offering a 100 -fold increase in recording speed for powder diffractometry. Designed for the X'Pert PRO diffractometer series, the $\mathrm{X}^{\prime}$ Celerator is based on the company's new real-time multiple-strip direction technology. This replaces a single detector with an integrated array of parallel detectors to provide up to 100 -fold increase in efficiency compared with traditional detectors. This means a scan formerly requiring three hours of data-collection time can now be recorded in less than two minutes, with no compromise on resolution.

The new unit is mounted using the company's proprietary PreFIX interface, a design that allows for easy exchange of

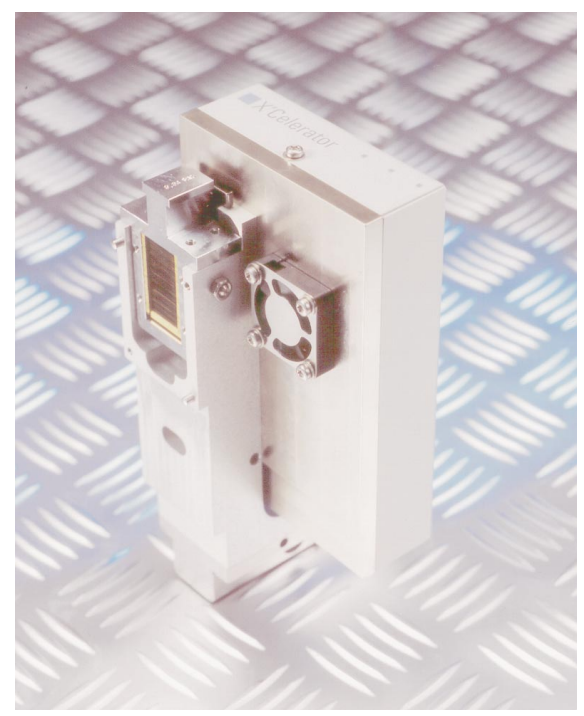

Figure 1

The Philips Analytical X'Celerator.

\title{
new commercial products
}

Announcements of new commercial products are published by the Journal of Applied Crystallography free of charge. The descriptions, up to 300 words or the equivalent if a figure is included, should give the price and manufacturer's full address. Full or partial inclusion is subject to the approval of the Editor, to whom all correspondence should be sent. The International Union of Crystallography can assume no responsibility for the accuracy of the claims made.

optical components without laborious realignment procedures. Its design makes for simple, maintenance-free operation. It is available with several options including a diffracted-beam monochromator, a beta filter and exchangeable Soller slits. With its high speed and ultra-high resolution, it is expected to offer advantages and cost savings in almost every powder-diffractometry application such as phase identification and quantification, crystallography and residual stress analysis.

\section{Philips Analytical}

Lelyweg 1

7602 EA Almelo

The Netherlands

E-mail: analytical.info@philips.com

Free ORTEC application note: dealing with dead-time distortion in a time digitizer

A new application note, AN57 Dealing with Dead Time Distortion in a Time Digitizer, is available free of charge from ORTEC. AN57 examines the dead-time distortions inherent with time digitizers applied to high event rates. Some of the results are somewhat surprising. The application note also includes a practical scheme for making deadtime corrections to the time spectrum after the spectrum has been acquired. The principles are elucidated by typical applications in time-of-flight mass spectrometry, although they are also applicable to LIDAR, optronics and nuclear spectrometry.

A free copy of AN57 can be ordered from info_ORTEC@perkinelmer.com, or downloaded from the application notes page of the ORTEC web site at http://www.ORTEConline.com.

\section{PerkinElmer Instruments}

801 South Illinois Ave

Oak Ridge

Tennessee 37830

USA

E-mail: info_ortec@perkinelmer.com

Free ORTEC application note: how histogramming and counting statistics affect peak-position precision

A new application note, AN58 How Histogramming and Counting Statistics Affect Peak Position Precision, is available free of charge from ORTEC. AN58 investigates and defines the relative importance of the errors caused by counting statistics, differential non-linearity, and bin width when determining the position of a peak in a spectrum that has been recorded as a histogram. This study is aimed at time digitizers, multichannel scalers and multichannel pulseheight analysers, where the counting of individual events follows Poisson statistics, but the results can be adapted to other instruments as well.

A free copy of AN58 can be ordered from info_ORTEC@perkinelmer.com, or downloaded from the application notes page of the ORTEC web site at http://www.ORTEConline.com.

\section{PerkinElmer Instruments}

801 South Illinois Ave

Oak Ridge

Tennessee 37830

USA

E-mail: info_ortec@perkinelmer.com 